\title{
Differential Intracochlear Sound Pressure Measurements in Normal Human Temporal Bones
}

\author{
Hideko Heidi Nakajima, ${ }^{1,2}$ Wei Dong, ${ }^{5}$ Elizabeth S. Olson, ${ }^{5}$ Saumil N. Merchant, ${ }^{1,2,4}$ \\ Michael E. Ravicz, ${ }^{1,2,3}$ and John J. Rosowski ${ }^{1,2,3,4}$ \\ ${ }^{1}$ Department of Otology and Laryngology, Harvard Medical School, Boston, MA 02115, USA \\ ${ }^{2}$ Eaton-Peabody Laboratory, Massachusetts Eye and Ear Infirmary, 243 Charles St., Boston, MA 02114, USA \\ ${ }^{3}$ Research Laboratory of Electronics, Massachusetts Institute of Technology, Cambridge, MA 02139, USA \\ ${ }^{4}$ Speech and Hearing Bioscience $\mathcal{E}$ Technology Program, Harvard-MIT Division of Health Sciences $\mathcal{E}$ Technology, Cambridge, \\ MA 02139, USA \\ ${ }^{5}$ Department of Otolaryngology, Head and Neck Surgery, Columbia University, P E S 11-452, 630 W. 168th Street, New York, \\ NY 10032, USA
}

Received: 23 April 2008; Accepted: 14 November 2008; Online publication: 9 December 2008

\begin{abstract}
We present the first simultaneous sound pressure measurements in scala vestibuli and scala tympani of the cochlea in human cadaveric temporal bones. The technique we employ, which exploits microscale fiberoptic pressure sensors, enables the study of differential sound pressure at the cochlear base. This differential pressure is the input to the cochlear partition, driving cochlear waves and auditory transduction. In our results, the sound pressure in scala vestibuli $\left(P_{\mathrm{SV}}\right)$ was much greater than scala tympani pressure $\left(P_{\mathrm{ST}}\right)$, except for very low and high frequencies where $P_{\mathrm{ST}}$ significantly affected the input to the cochlea. The differential pressure $\left(P_{\mathrm{SV}}-P_{\mathrm{ST}}\right)$ is a superior measure of ossicular transduction of sound compared to $P_{\mathrm{SV}}$ alone: $\left(P_{\mathrm{SV}}-P_{\mathrm{ST}}\right)$ was reduced by 30 to $50 \mathrm{~dB}$ when the ossicular chain was disarticulated, whereas $P_{\mathrm{SV}}$ was not reduced as much. The middle ear gain $P_{\mathrm{SV}} / P_{\mathrm{EC}}$ and the differential pressure normalized to ear canal pressure $\left(P_{\mathrm{SV}}-P_{\mathrm{ST}}\right) / P_{\mathrm{EC}}$ were generally bandpass in frequency dependence. At frequencies above $1 \mathrm{kHz}$, the group delay in the middle ear gain is about $83 \mu$ s, over twice that of the gerbil. Concurrent measurements of stapes velocity produced estimates
\end{abstract}

Correspondence to: John J. Rosowski · Eaton-Peabody Laboratory · Massachusetts Eye and Ear Infirmary · 243 Charles St., Boston, MA 02114, USA. Telephone: +1-617-4130616; fax: +1-617-7204408; email: John_Rosowski@meei.harvard.edu of cochlear input impedance, the differential impedance across the partition, and round window impedance. The differential impedance was generally resistive, while the round window impedance was consistent with compliance in conjunction with distributed inertia and damping. Our technique of measuring differential pressure can be used to study inner ear conductive pathologies (e.g., semicircular dehiscence), as well as non-ossicular cochlear stimulation (e.g., round window stimulation and bone conduction) - situations that cannot be completely quantified by measurements of stapes velocity or scala vestibuli pressure by themselves.

Keywords: middle ear, cochlea, cochlear impedance, scala vestibuli, scala tympani, stapes velocity

\section{INTRODUCTION}

Many quantities need to be measured to understand the mechanical and acoustical processes in the middle and inner ears. Traditional measurements of middle ear function, such as stapes velocity, are useful for studying sounds transmitted by air conduction in normal ears and ears with middle ear lesions. However, the study of inner ear conductive pathologies (e.g., semicircular canal dehiscence; Merchant 
and Rosowski 2008) and the stimulation of the cochlea by non-ossicular means (e.g., round window stimulation, Colletti et al. 2006; bone conduction) require a different approach: differential pressure measurements. We describe the first differential pressure measurements in human cadaveric temporal bones. These measurements are made possible by microscale pressure sensors developed by Olson (1998) which enable simultaneous sound pressure measurements in scala vestibuli and scala tympani. The measurements reported in this study provide a baseline for future measurements to study various middle and inner ear pathologies as well as the effect of non-ossicular cochlear stimulation.

Sound propagates down the ear canal and vibrates the tympanic membrane, resulting in vibration of the middle ear ossicles. The vibration of the stapes in the oval window produces sound pressure within the vestibule of the cochlea resulting in translation of the cochlear fluid. Across the cochlear partition from the vestibule, the sound pressure is relieved by the compliant round window in scala tympani, with a resulting pressure difference across the partition. This differential intracochlear pressure across the base of the cochlear partition is the input signal to the cochlea and starts the traveling wave moving toward the apex. The pressure difference thus drives auditory transduction.

Dancer and Franke (1980) and Lynch et al. (1982) demonstrated that the differential sound pressure measured in the guinea pig and cat, respectively, closely follows that of the cochlear microphonic measured in a similar location. Voss et al. (1996) also showed that the pressure difference between the oval and round windows is the stimulus that produces cochlear responses in the cat. This supports the idea that the differential sound pressure measured at the base of the cochlea is the input signal for the transduction of sound by the cochlea.

In this study, we measured the sound-evoked differential pressure at the base of the cochlea in human temporal bones with two microscale pressure sensors: one in scala vestibuli near the oval window and one in scala tympani near the round window. The sensitive tips of the pressure sensors were located within $200 \mu \mathrm{m}$ of the bony wall of the cochlea and relatively distant from the cochlear partition. Hence, the pressures measured simultaneously by the probes in our experiments were relatively unaffected by the near-field pressures of the cochlear partition that result from the motion of the basilar membrane, as compared to measurements made close to the partition (Olson 1998).

This new technique in the fresh human temporal bone allows for the study of how the input to cochlear transduction (differential pressure across the parti- tion) is affected by various middle and inner ear pathologies and modifications. Examples of pathologies include ossicular discontinuity as well as cochlear fistulas ("third window") such as superior semicircular canal dehiscence, known to result in conductive hearing loss (Merchant and Rosowski 2008). Furthermore, the effect of stimulating the cochlea in an unconventional manner can be studied. Examples of unconventional cochlear stimulation include mechanical stimulation of the round window (which has been performed on patients for various mixed hearing losses, Colletti et al. 2006), as well as bone conduction. Therefore, these measurements provide a baseline for comparison with future measurements to answer clinically relevant questions.

In this manuscript, we describe simultaneous pressure measurements in scala vestibuli and scala tympani in normal cadaveric ears. Comparisons are made to previous scala vestibuli measurements in human cadavers as well as to differential pressure measurements in other species. The effect of ossicular discontinuity on intracochlear pressures is also described. Furthermore, measurements of stapes velocity are made concurrently, allowing for the calculation of the cochlear input impedance, the differential impedance across the partition, and the round window impedance. Obtaining these impedances is important for understanding how the cochlea transduces sound pressure from the middle ear to the cochlea.

\section{METHODS}

\section{Temporal bone preparation}

This study was approved by the Institutional Review Board of the Massachusetts Eye and Ear Infirmary. Fourteen human temporal bones were used for this study. The first seven were used to perfect the technique. Of the remaining seven temporal bones, the results from six, which did not appear to have intracochlear air and showed stable pressure probe calibrations, are presented. The bone that is not included showed changes in stapes and round window velocities after pressure sensor insertions. Temporal bones were obtained from cadavers that were undergoing autopsy with permission to use tissues and organs for research. Removal of temporal bones was performed by an intracranial approach using a Schuknecht plug cutter by the method described in Nadol (1996). The specimens were stored in 0.9\% normal saline and refrigerated immediately upon removal. Surrounding soft tissue was removed and the medial and posterior aspects of the petrous bone were sealed with dental cement to prevent the introduction of air into the cochlea and loss of 
intracochlear fluid. The bony ear canal was shortened to a length of about $1 \mathrm{~cm}$, and the facial recess was opened for access to the middle and inner ears. The stapedial tendon was removed to allow access to the area surrounding the oval window. The promontory was thinned near the oval and round windows where the pressure probes were to be inserted. The temporal bone was positioned such that the locations of the pressure-sensor insertions were uppermost relative to the rest of the bone to prevent entry of air into the cochlea when the cochleostomies were made and the pressure sensors were inserted.

\section{Sound stimulation, recording at the ear canal, and signal processing}

Tones between $100 \mathrm{~Hz}$ and $20 \mathrm{kHz}$ (61 frequencies) and 70-130 dB SPL were presented with a Radio Shack driver (400-1377) connected by flexible tubing to a sealed bony ear canal. To record ear canal sound near the tympanic membrane, a calibrated Etymotic microphone (ER-7C) flexible probe tube tip was positioned approximately 1 to $3 \mathrm{~mm}$ from the umbo. Sound was presented for sound-evoked intracochlear measurements and stapes and round window velocity measurements. These measurements were checked to confirm linearity for the sound pressure range used.

Pressure measurements in the scala vestibuli and scala tympani were made simultaneously. Stapes and round window velocity and sound pressure in the ear canal were also measured. The two channels available were used to make pair-wise simultaneous measurements, and sound pressure measurements were checked before and after intracochlear pressure measurement sequences to confirm stability. Segments of measurements, $20.4 \mathrm{~ms}$, were sampled at $226 \mathrm{kHz}$ and averaged 50 times. Spectra were computed from the averaged waveforms by Fourier analysis. Statistics of the data were calculated by taking the geometric mean and standard deviation of the magnitude and the arithmetic mean and standard deviation of the phase of the stimulus-frequency component. All measurements plotted are for responses with at least $10 \mathrm{~dB}$ signal-to-noise ratio unless otherwise specified.

\section{Velocity measurements}

A laser Doppler vibrometer system (Polytec CLV 700) aimed at an approximately $0.2 \mathrm{~mm}^{2}$ reflector (consisting of $50 \mu \mathrm{m}$ diameter reflective polystyrene microbeads on plastic tape) applied to the posterior crus, round window or cochlear promontory, was used for velocity measurements. Laser velocity measurements were made of the stapes posterior crus from an angle about $45^{\circ}$ to $60^{\circ}$ with respect to the footplate.
Velocity measurements were also made of the round window membrane. A phase difference between the stapes and round window of one-half cycle at frequencies below $500 \mathrm{~Hz}$, before and after cochleostomies and pressure probe insertions, was used as confirmation that air was not introduced into the cochlea (Kringlebotn 1995; Stenfelt et al. 2004a, b). Both magnitude and phase of the stapes and round window velocities changed if air was introduced by the insertions of the pressure sensors. To measure "artifact" and "noise" for ossicular velocity measurements, we measured the vibration of the temporal bone at the cochlear promontory induced by the same acoustic stimuli used for the ossicular velocity measurements (Nakajima et al. 2005).

\section{Pressure sensor}

Intracochlear pressure measurements were performed with microoptical pressure sensors developed by Olson (1998). The advantage of these pressure sensors is their small size of $167 \mu \mathrm{m}$ diameter and their frequency-independent sensitivity to $40 \mathrm{kHz}$. Details regarding their design and fabrication can be found in Olson (1998). Briefly, the tip of the pressure sensor is made of a glass microtube terminated on one end by a thin plastic film diaphragm coated with a reflective layer of evaporated gold. Inserted into the open end of the microtube is an optical fiber positioned a small distance $(50-100 \mu \mathrm{m})$ from the diaphragm tip. Light from the optical fiber is split into two fibers at its other end, with one attached to a LED light source and the other to a photodiode sensor. Light from the LED source reaches the sensor tip of the optical fiber and is reflected by the gold-covered flexible diaphragm. This reflected light is sensed by the photodiode. Small sound-induced displacements of the diaphragm modulate the reflected light intensity, imparting a sensitivity to sound. The output of the sensor varies linearly over a wide range of sound pressure.

The pressure sensors were calibrated in water using a shaker (Bruel and Kjaer 4290) with an internal accelerometer whose output was cross-checked by measuring its velocity with laser Doppler vibrometry. Details of the calibration method can be found in Nedzelnitsky (1980) and Schloss and Strasberg (1962). In initial experiments, calibrations performed in water and in air were found to agree. The sensitivities of the sensors varied between -155 and $-140 \mathrm{~dB}$ re $1 \mathrm{~V} / 20 \mu \mathrm{Pa}$. Before an experiment, several pressure sensors were fabricated, tested for stability, and calibrated in water. Because the sensitivities of the pressure sensors are prone to change due to mechanical perturbation, measurement sequences were kept short to less than approximately $2 \mathrm{~h}$. To ensure 
accuracy of the pressure sensors, for each sequence of measurements, the pressure sensors were calibrated in water just prior to insertion and immediately after removal from the scala. Only measurements with pressure sensor calibrations that were stable to within $2 \mathrm{~dB}$ are presented.

Accessing the inner ear for intracochlear pressure measurements

Cochleostomies just slightly larger than the pressure sensor diameter (with holes approximately $200 \mu \mathrm{m}$ for the $167-\mu \mathrm{m}$ sensors) were drilled by hand while the cochlea was immersed in saline solution to prevent introduction of air into the cochlea. The pressure sensors were inserted into scala vestibuli and scala tympani, approximately $200 \mu \mathrm{m}$ into the scala from the surface of the cochlea. The saline surrounding the cochlea was removed and the gaps between the pressure sensors and the bone were sealed with dental impression material (Jeltrate, L.D. Caulk Co.), which dried to a rubbery consistency. Figure $1 \mathrm{~B}$ shows the preparation with the pressure probes inserted into scala vestibuli and scala tympani and their surrounding Jeltrate seals.

At the end of the experiment, the positions of the pressure sensors were confirmed by opening the round window membrane and the surrounding bone over scala tympani and scala vestibuli. The partition was carefully examined for possible trauma. Figure 1C shows a preparation after an experiment where the hole used for scala tympani pressure probe and the notch from the hole in scala vestibuli can be seen. Inspection demonstrates that the pressure probes were inserted in the scala vestibuli and tympani. The partition and the basilar membrane can be seen through the opened round window area. Figure 1A illustrates the locations of various measurements performed: the ear canal pressure $\left(P_{\mathrm{EC}}\right)$ was measured near the umbo with a probe tube, stapes velocity $\left(V_{\text {Stap }}\right)$ and round window velocity $\left(V_{\mathrm{RW}}\right)$ were measured on the posterior crus and center of the round window membrane, respectively, and the scala vestibuli pressure $\left(P_{\mathrm{SV}}\right)$ and scala tympani pressure $\left(P_{\mathrm{ST}}\right)$ were measured near the stapes and round window, respectively.

\section{RESULTS}

\section{Transfer functions of intracochlear pressures}

The relationship between the intracochlear pressures (both scala vestibuli and scala tympani) and the ear canal pressures (between 80 and $130 \mathrm{~dB}$ SPL) were linear and stable with time. The magnitude and phase of level-independent transfer func- tions from ear canal pressures to scala pressures for six temporal bones are illustrated in Figure 2. The frequency responses for the scala vestibuli transfer function $\left(P_{\mathrm{SV}} / P_{\mathrm{EC}}\right)$ are plotted in the left panels (A) and the scala tympani $\left(P_{\mathrm{ST}} / P_{\mathrm{EC}}\right)$ in the right panels (B). There was much similarity between the pressures measured in the six ears, and there were large differences between the frequency responses of the pressure in scala vestibuli and scala tympani in each ear. One ear, 039, had higher $P_{\text {SV }}$ pressures at frequencies above $1 \mathrm{kHz}$ and at very low frequencies below $300 \mathrm{~Hz}$, as well as higher $P_{\mathrm{ST}}$ pressure at frequencies below $400 \mathrm{~Hz}$.

The average and standard deviation of the transfer functions of scala vestibuli and scala tympani pressures relative to the ear canal pressure are plotted together in Figure 3. The pressure in scala vestibuli was generally 10 to $20 \mathrm{~dB}$ larger in magnitude than in scala tympani over a wide range of frequencies. For frequencies below $500 \mathrm{~Hz}$, the phase of the scala tympani pressure relative to ear canal pressure was slightly less than zero while the phase of the scala vestibuli pressure had almost $1 / 8$ cycle lead. Above $500 \mathrm{~Hz}$, the phases of the pressures within both scalae were generally similar.

\section{Pressure gain of the middle ear}

The ratio of sound pressure in scala vestibuli to that of the ear canal represents the pressure gain of the middle ear. Figure 4 compares the mean and standard deviation of the middle ear pressure gain $\left(P_{\mathrm{SV}} / P_{\mathrm{EC}}\right)$ between Aibara et al. $(2001, n=11)$ and the present study $(n=6)$. Over most frequencies, the results from the two studies were similar. The gain was bandpass in nature, rising at low frequencies and falling off above $3 \mathrm{kHz}$. The maximum middle ear pressure gain was approximately $20 \mathrm{~dB}$ near $1 \mathrm{kHz}$. The individual data from the present study tended to show a decrease in magnitude between 5 and $7 \mathrm{kHz}$, which when averaged resulted in a "notch" in that frequency region. Aibara et al. (2001) also had notches in the individual recordings, but the frequency of the notches varied across ears between 3 and $8 \mathrm{kHz}$ and cancelled with averaging. The phases of the two studies were similar.

\section{Middle ear transmission delay}

Figure 5 plots the same data as in Figure 4 (average magnitude and phase of the middle ear pressure gain) on a linear frequency scale. While the magnitude was bandpass in nature, the phase fell proportionally with frequency above approximately $1 \mathrm{kHz}$, consistent with the middle ear having properties of a simple delay with an estimated group delay of $83 \mu$ s. 

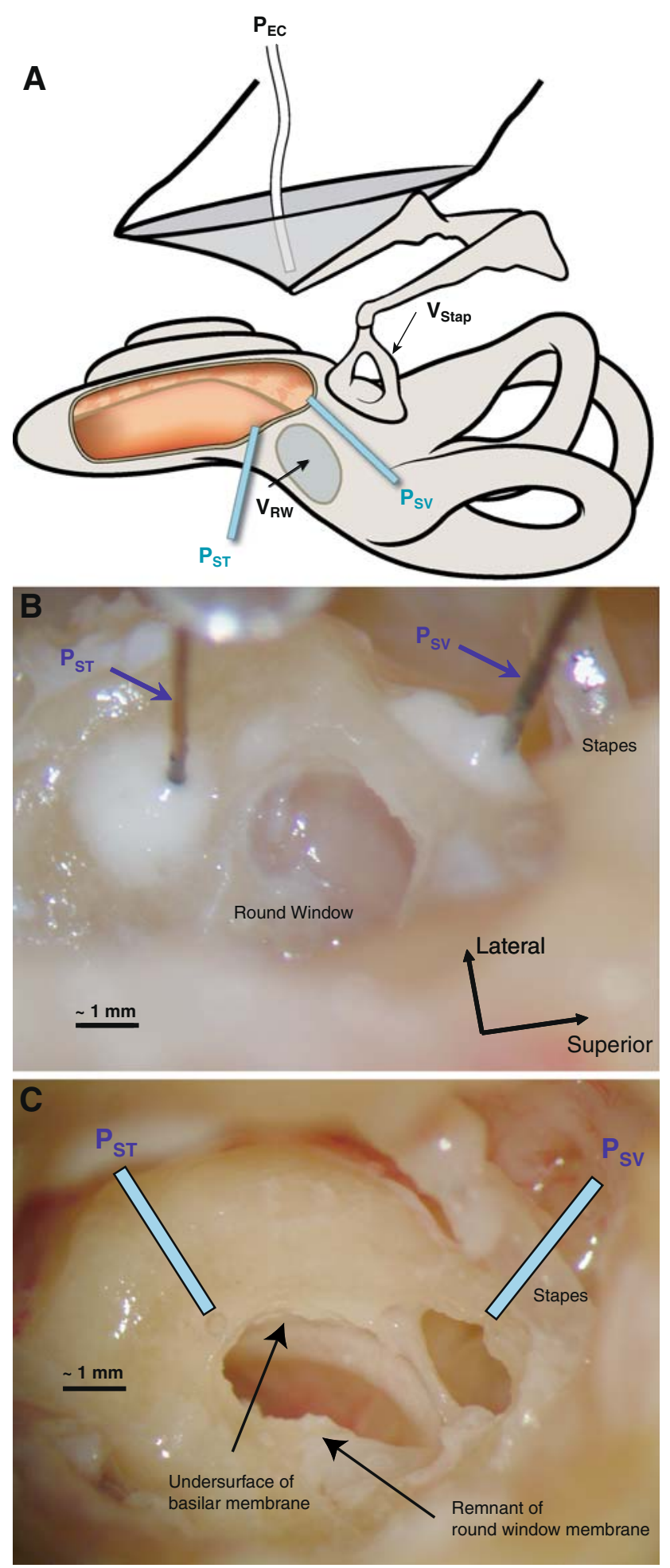

\section{Differential pressure}

The differential pressure is defined as the complex difference between scala vestibuli and scala tympani sound pressures measured near the cochlear walls in the base of the cochlea. The cochlear differential
FIG. 1. A Illustration showing the locations of various types of recordings: pressure in scala vestibuli $\left(P_{\mathrm{SV}}\right)$, pressure in scala tympani $\left(P_{\mathrm{ST}}\right)$, pressure in the ear canal $\left(P_{\mathrm{EC}}\right)$, velocity of the stapes $\left(V_{\mathrm{Stap}}\right)$, velocity of the round window $\left(V_{\mathrm{RW}}\right)$. B Photograph of left temporal bone showing pressure sensors inserted into scala vestibuli $\left(P_{\mathrm{SV}}\right)$ and scala tympani $\left(P_{\mathrm{ST}}\right)$. Jeltrate seals the holes surrounding the pressure probes. C Photograph of post-experimental preparation that has been opened to verify location of sensors. The hole used for scala tympani pressure sensor $\left(P_{\mathrm{ST}}\right)$ and the notch from the hole for scala vestibuli pressure sensor $\left(P_{\mathrm{SV}}\right)$ can be seen. The cochlear partition and the basilar membrane can be seen through the opened round window area.

pressure, normalized by the ear canal pressure near the tympanic membrane, $\left(P_{\mathrm{SV}}-P_{\mathrm{ST}}\right) / P_{\mathrm{EC}}$, is plotted in Figure 6 for six temporal bones. Because $\left|P_{\mathrm{ST}}\right|$ was generally smaller than $\left|P_{\mathrm{SV}}\right|$ (Fig. 3), the normalized differential pressure was similar to the middle ear gain in the mid-frequency region.

\section{Effect of disrupting the ossicular chain on intracochlear pressures}

We disrupted the ossicular chain by disarticulating the incudostapedial joint and removing the lenticular process, producing an air gap between the head of the stapes and incus. The effect of this manipulation on the intracochlear pressures is plotted in Figure 7. After disrupting the ossicular chain, there were large (20 to $40 \mathrm{~dB}$ ) decreases in (A) scala vestibuli and (B) scala tympani pressures for frequencies below $5 \mathrm{kHz}$. At higher frequencies, the decreases were smaller than $20 \mathrm{~dB}$ and were only a few $\mathrm{dB}$ at select frequencies. The significant sound pressures measured at certain frequencies (e.g., $6 \mathrm{kHz}$ ) after ossicular interruption suggest that sound is transmitted to both scalae through a path independent of the ossicular chain. The phase after ossicular interruption is shifted by $180^{\circ}$ compared to the intact middle ear.

Figure 8 plots the differential pressure relative to the ear canal pressure, $\left(P_{\mathrm{SV}}-P_{\mathrm{ST}}\right) / P_{\mathrm{EC}}$, before and after severing the ossicular chain. Ossicular discontinuity results in $30-50 \mathrm{~dB}$ decrease in the differential pressure over a wide frequency range, demonstrating the cancellation of similar sound pressures in both scalae.

\section{DISCUSSION}

This study describes the first intracochlear measurements of differential pressure in human cadaveric temporal bones. This differential pressure represents the input signal to the cochlea, which produces partition motion, resulting in the traveling wave moving toward the apex, resulting in auditory signal transduction. We now have a method by which we can 

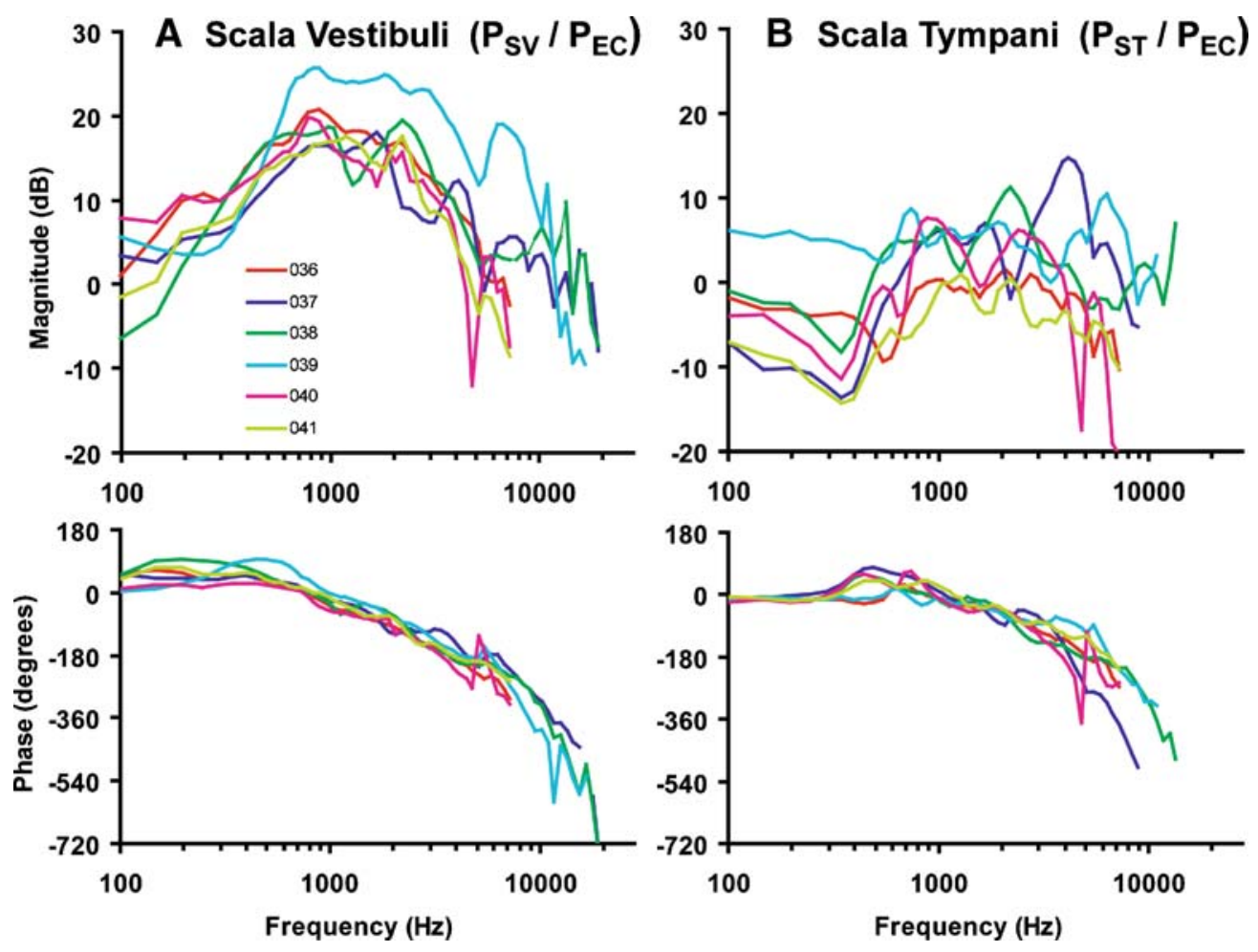

FIG. 2. Sound pressure (magnitude and phase) in A scala vestibuli and B scala tympani, normalized to the ear canal sound pressure, for six temporal bones.

detect the effect of middle ear and inner ear modifications as well as various methods of sound input to the cochlea on the input signal to the cochlea.

\section{Pressure measurements in cochlear scalae}

Intracochlear pressure in scala vestibuli and scala tympani at the base of the cochlea was measured close to the cochlear surface $(200 \mu \mathrm{m})$ and relatively far away (2 to $3 \mathrm{~mm}$ ) from the partition. This enabled us to measure the overall input to the cochlea that would initiate the traveling wave at the partition. These pressures measured near the surface of the cochlea are little affected by the local pressure changes due to a traveling wave that are seen in pressures measured near the basilar membrane (Olson 1998, 1999). The magnitude of scala vestibuli pressure was much larger than that in scala tympani for a wide range of frequencies, except at low (below $300 \mathrm{~Hz}$ ) and high (above $5 \mathrm{kHz}$ ) frequencies (Fig. 3). Therefore, the scala tympani pressure has a significant effect on the pressure difference at low and high frequencies. The scala tympani pressure phase was near zero while the scala vestibuli pressure phase showed a lead of near $1 / 8$ cycle at low frequencies (below $500 \mathrm{~Hz}$ ). The phases were generally similar at higher frequencies. Similar findings were reported in cat (Nedzelnitsky 1980) and the guinea pig (Dancer and Franke 1980).
Pressure gain of the middle ear

Scala vestibuli sound pressure can be regarded as the output of the middle ear, and the $P_{\mathrm{SV}} / P_{\mathrm{EC}}$ transfer function gives insight regarding the pressure gain produced by the middle ear. The pressure gain from the tympanic membrane to the vestibule was bandpass in nature with a maximum magnitude of approximately $20 \mathrm{~dB}$ near $1 \mathrm{kHz}$ (Fig. 4). Below $1 \mathrm{kHz}$, the slope of the magnitude was approximately $20 \mathrm{~dB} /$ decade and the phase was approximately $1 / 8$ cycle, indicating that the elastic properties of middle ear components (tympanic membrane, middle ear ligament, middle ear joints, annular ligament) dominate, reducing the middle ear gain. Above $3 \mathrm{kHz}$, the magnitude of the gain fell off at about $-20 \mathrm{~dB}$ per decade, and the phase accumulated nearly two cycles of delay between 2 and $20 \mathrm{kHz}$.

Previous studies have also measured cadaveric human middle ear pressure gain - the ratio of the pressures between the vestibule near the footplate and the ear canal near the tympanic membrane $\left(P_{\mathrm{SV}} / P_{\mathrm{EC}}\right)$. Puria et al. (1997) made pressure measurements on four ears with a hydrophone pressure transducer, while keeping a flow of fluid through tubing connected to the superior semicircular canal and through tubing connected near the round window to flush possible air that might accumulate. Later, Aibara et al. (2001) made similar measure- 

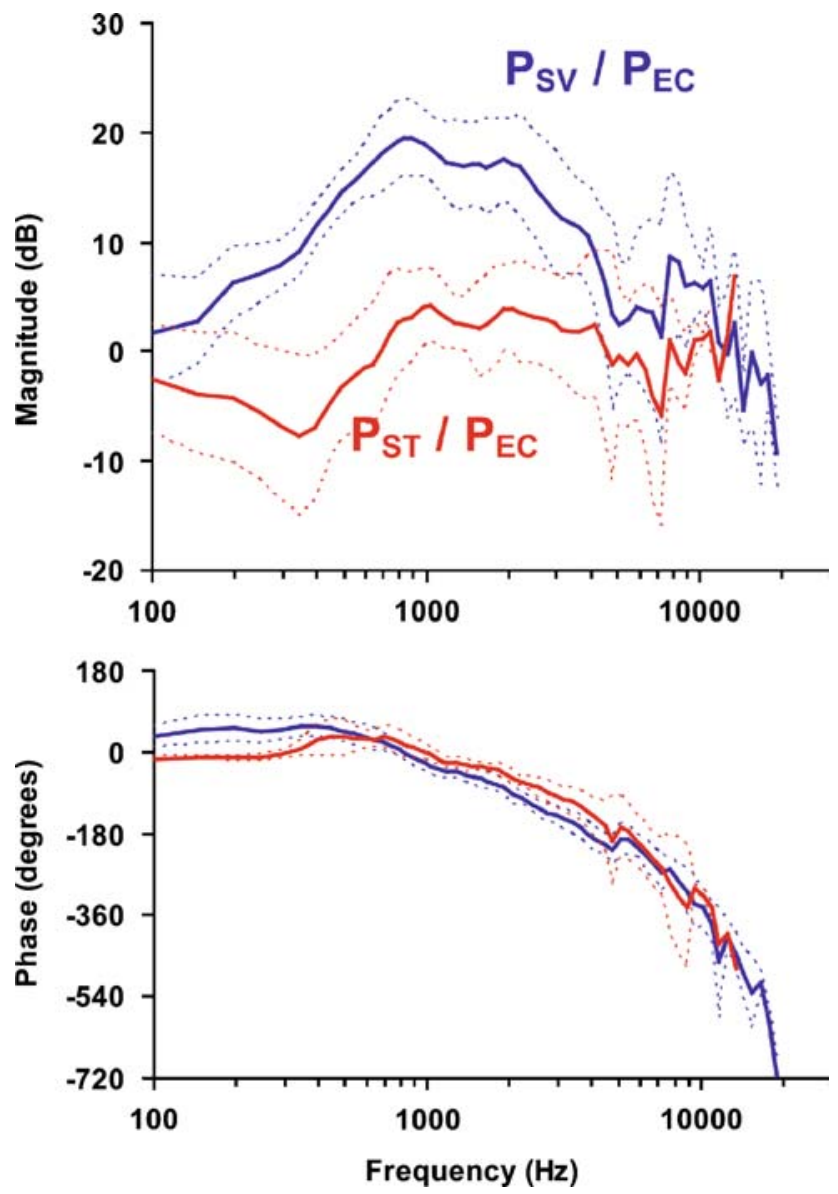

FIG. 3. The means and standard deviations of the pressures in scala vestibuli and scala tympani relative to the ear canal pressure.

ments in 11 ears near the footplate by inserting a 1.4-mm diameter hydrophone pressure transducer through an opening near the singular canal while under saline to prevent introduction of air into the cochlea. The methods of Aibara et al. (2001) are more similar to our methods (in that there is no artificial flow of fluid through tubings); however, Aibara et al. made their measurement from the medial aspect while we made our measurement from the lateral aspect. Despite the different pressure transducers and approach to the vestibule, the middle ear pressure gain $\left(P_{\mathrm{SV}} / P_{\mathrm{EC}}\right)$ is similar among all three studies (Fig. 4 comparing our measurements to Fig. 6 of Aibara et al. 2001).

\section{Middle ear transmission delay}

By plotting the pressure gain of the middle ear on a linear frequency scale, we can estimate the group delay of the gain by identifying regions of linear relationship between the phase and frequency (Fig. 5). Our data suggest a group delay of $83 \mu$ s at frequencies above $1 \mathrm{kHz}$. This is identical to the group delay calculated from Puria (2003) data by
Dong and Olson (2006). This delay is more than twice the 25- to 32- $\mu$ s delay found in the gerbil (Olson 1998; Dong and Olson 2006). The increased delay observed in human temporal bones is likely related to interspecies differences in transduction from the ear canal to the inner ear. While others have observed that the amplitude and phase of middle ear transmission in other species suggests that the middle ear acts as a lossless transmission line (Puria and Allen 1998; Olson 1998; Ruggero and Temchin 2002), the roll off in magnitude in the human middle ear gain above $2 \mathrm{kHz}$ is not consistent with a lossless middle ear model. A possible explanation is that humans have larger effective ossicular mass, or higher ossicular joint compliance (Willi et al. 2002; Nakajima et al. 2005) and/or a more compliant tympanic membrane compared to other mammals.

\section{Differential pressure}

The intracochlear sound pressure difference across the cochlear partition $\left(P_{\mathrm{SV}}-P_{\mathrm{ST}}\right)$ at the base of the cochlea is the input force that drives the motion of the cochlear partition, setting off the traveling wave. In the mid-frequencies where $\left|P_{\mathrm{SV}}\right|$ is significantly
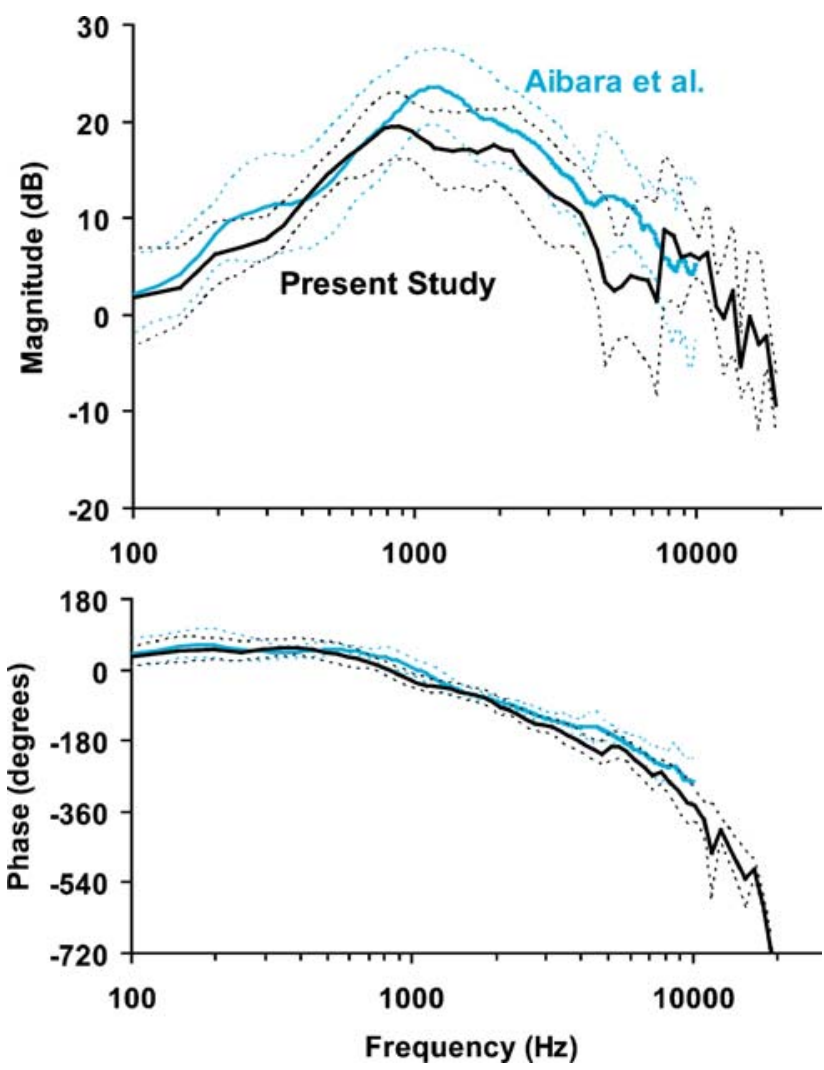

FIG. 4. Comparison of the mean and standard deviation of the middle ear pressure gain $\left(P_{\mathrm{SV}} / P_{\mathrm{EC}}\right)$ between Aibara et al. $(n=11$, 2001) and the present study $(n=6)$. 


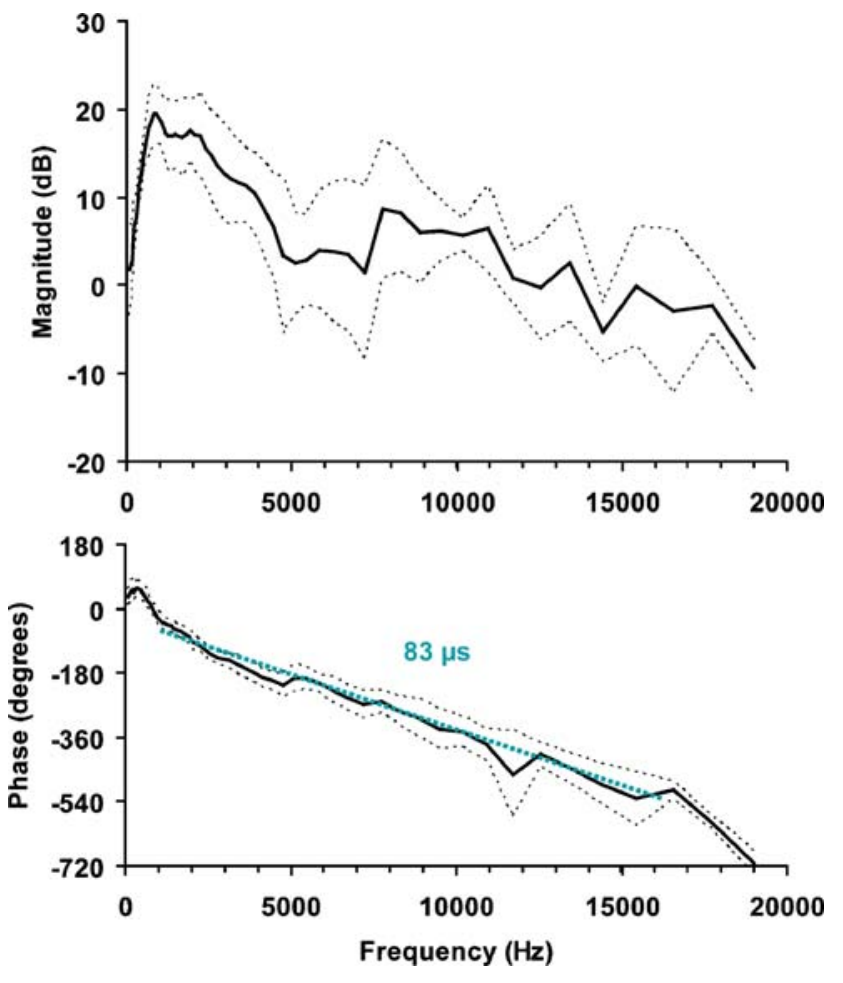

FIG. 5. This figure plots the same average magnitude and phase of our middle ear pressure gain as in Figure 4, on a linear scale. The linear phase above $1 \mathrm{kHz}$ is consistent with a group delay of $83 \mu \mathrm{s}$.

greater than $\left|P_{\mathrm{ST}}\right|$, the differential pressure is dominated by $P_{\mathrm{SV}}$ (Figs. 2 and 3). At low and high frequencies, the differential input pressure is significantly affected by the scala tympani pressure because the latter is similar in magnitude to the scala vestibuli pressure.

The differential pressure at the cochlear base in humans (mean and standard deviation, $n=6$ ) from the present study is plotted in Figure 9 with that of the cat (median, $n=6$, Nedzelnitsky 1980) and the guinea pig (mean, $n=5$, Dancer and Franke 1980). Compared to these animals, the basal differential pressure of humans has a smaller magnitude with a clear highfrequency cutoff with increased phase lag at high frequencies (Fig. 9). It should be noted that one ear of our six had a higher differential gain in the high frequencies (Fig. 6). The significance of this one measurement is unknown. The cat and human data are similar, with a peak magnitude near $1 \mathrm{kHz}$ but the magnitude of the pressure difference is larger in cat. The cat can hear up to approximately $60 \mathrm{kHz}$, yet the data available are limited to $7 \mathrm{kHz}$, therefore the highfrequency trend is unknown. The guinea pig differential pressure data are similar to the cat and human up to approximately $1 \mathrm{kHz}$, but continues to increase in magnitude with a peak near $10 \mathrm{kHz}$. Futhermore, the phase in the cat and guinea pig lags less at high frequency compared to the human.
Effect of ossicular discontinuity on sound transmission

The forward transmission of sound in the normal ear is assumed to be through the ear canaltympanic membrane-ossicular chain-cochlea. To determine whether the measured intracochlear sound pressures are produced solely by ossicular transmission, we disrupted the ossicular chain. This manipulation was also a check for artifacts in our preparation, stimulus, and recording system. It allows us to determine whether our measurement is recording the effects of ossicular sound transmission or whether there are other means of sound transmission to the cochlea. Furthermore, when ossicular discontinuity occurs clinically, it results in a large ( 40 to $60 \mathrm{~dB}$ ) conductive hearing loss throughout all frequencies.

Disarticulating the ossicular chain substantially decreased (over $20 \mathrm{~dB}$ ) sound pressure in both scala vestibuli and scala tympani for low frequencies (below $5 \mathrm{kHz}$ ), but significant sound pressure was observed at higher frequencies in both scalae (Fig. 7). Similar results were recorded by Puria et
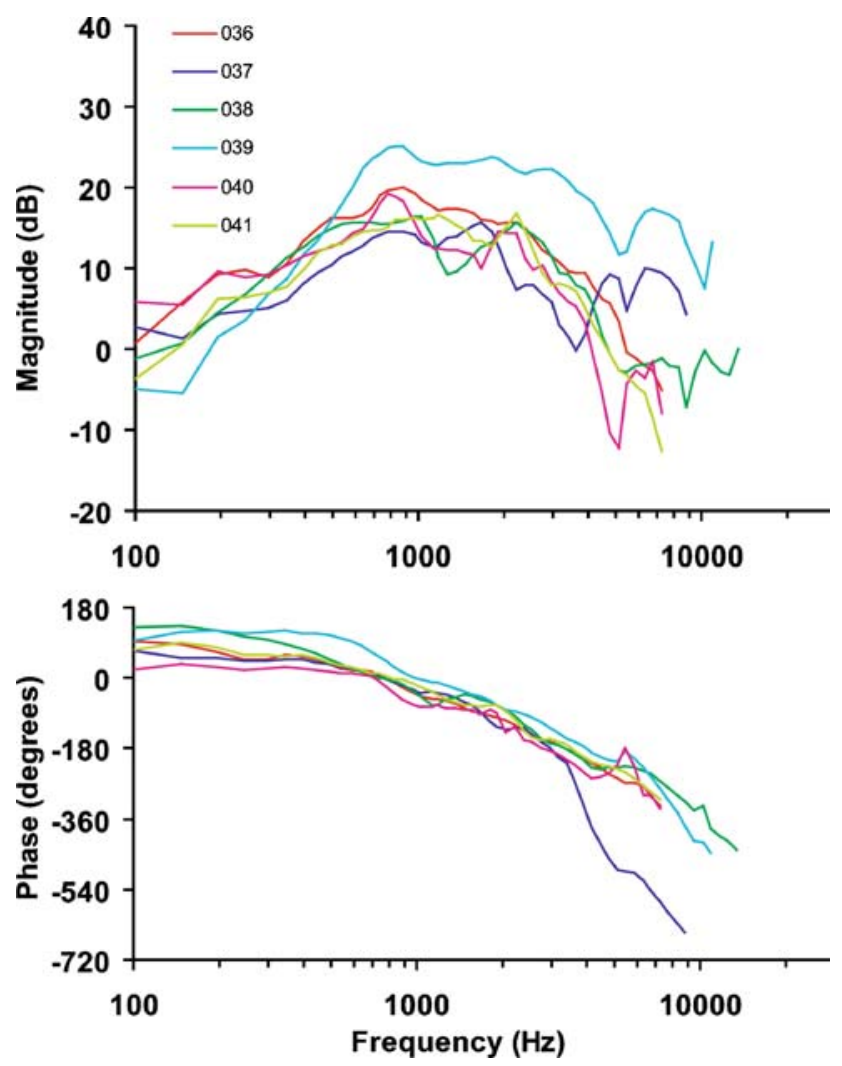

FIG. 6. Computed differential pressure, defined as the complex pressure difference across the partition normalized to ear canal pressure, $\left(P_{\mathrm{SV}}-P_{\mathrm{ST}}\right) / P_{\mathrm{EC}}$, for six temporal bones. This difference in pressure at the base of the cochlea represents the input signal to the cochlea. 

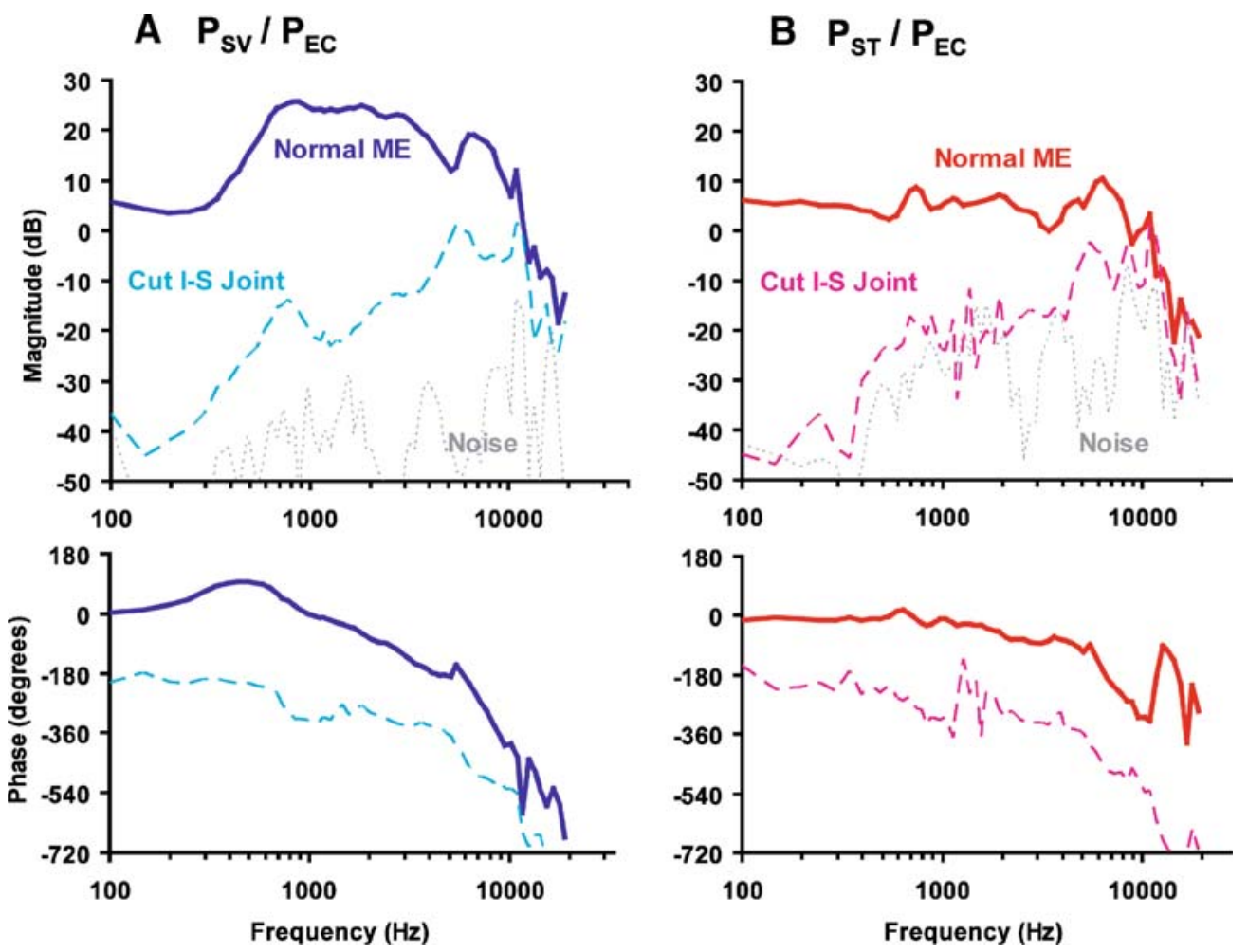

FIG. 7. An example (ear 039) of effects due to disrupting the ossicular chain. After disrupting the ossicular chain, there are large (20-40 dB) decreases in A scala vestibuli and $\mathbf{B}$ scala tympani pressures for frequencies $<5 \mathrm{kHz}$. At higher frequencies, the decreases are smaller (10-20 dB), demonstrating conducted sound to both scalae without an intact middle ear.

al. (1997) in scala vestibuli in human temporal bones as well as by Nedzelnitsky (1980) for both scalae in the cat. Close inspection of our data shows that this non-ossicularly conducted sound appears nearly equally in scala vestibuli and scala tympani. A possible source for this sound without an intact ossicular chain is shaking of the entire bone by the coupled sound source.

Because the difference in pressure across the partition is the measure most relevant to cochlear stimulation, the effect of severing the ossicular chain on the differential input pressure was also quantified (Fig. 8). Ossicular discontinuity resulted in large $(30-50 \mathrm{~dB})$ decreases in the differential pressure for most frequencies, demonstrating the cancellation of similar non-ossicularly conducted sound signals in both scalae. Thus, the differential input pressure was greatly affected by interruption of the ossicular chain, demonstrating that the differential pressure measurement is a superior measure for studying auditory pathology such as ossicular discontinuity, compared to pressure measurement in scala vestibuli alone. The large 30 $50 \mathrm{~dB}$ drop in differential pressure across a wide frequency range is consistent with the conductive hearing loss seen in patients as a result of ossicular discontinuity (Merchant and Rosowski 2003).

\section{Cochlear input impedance $\left(Z_{C}\right)$}

The transmission of sound to the cochlea via the middle ear can be understood by the relationship between force and motion at the oval window. The load on the middle ear imposed by the cochlea at the oval window can be characterized by the cochlear input impedance $\left(Z_{C}\right)$ with units of acoustic impedance, mks $\Omega$ (Pa s/ $\mathrm{m}^{3}$ ) (Zwislocki 1975). Because we measured the stapes velocity concurrently with scala pressures, we can estimate $Z_{\mathrm{C}}$,

$$
Z_{C}=P_{S V} / U_{S t a p}
$$

where the volume velocity $\left(U_{\text {Stap }}\right)$ of the stapes footplate is equal to the stapes velocity times the area of the footplate. We use $3.2 \mathrm{~mm}^{2}$ as the area of the footplate, consistent with the estimates of von Békésy (1960) and Aibara et al. (2001). Thus $Z_{\mathrm{C}}$ is the ratio between $P_{\mathrm{SV}}$ (referenced to the open middle ear space equal to zero) and $U_{\text {stap }}$. The average and standard deviation of the calculated cochlear input impedance, $Z_{C}$, is plotted in Figure 10. $\angle Z_{C}$ is approximately $0^{\circ}$ up to about $5 \mathrm{kHz}$. This is consistent with the cochlear input impedance behaving generally as an acoustic resistance. Unlike a pure resistance, $\left|Z_{C}\right|$ varies somewhat with frequency. Our present study $(n=6)$ results in values of $Z_{C}$ that generally fall 

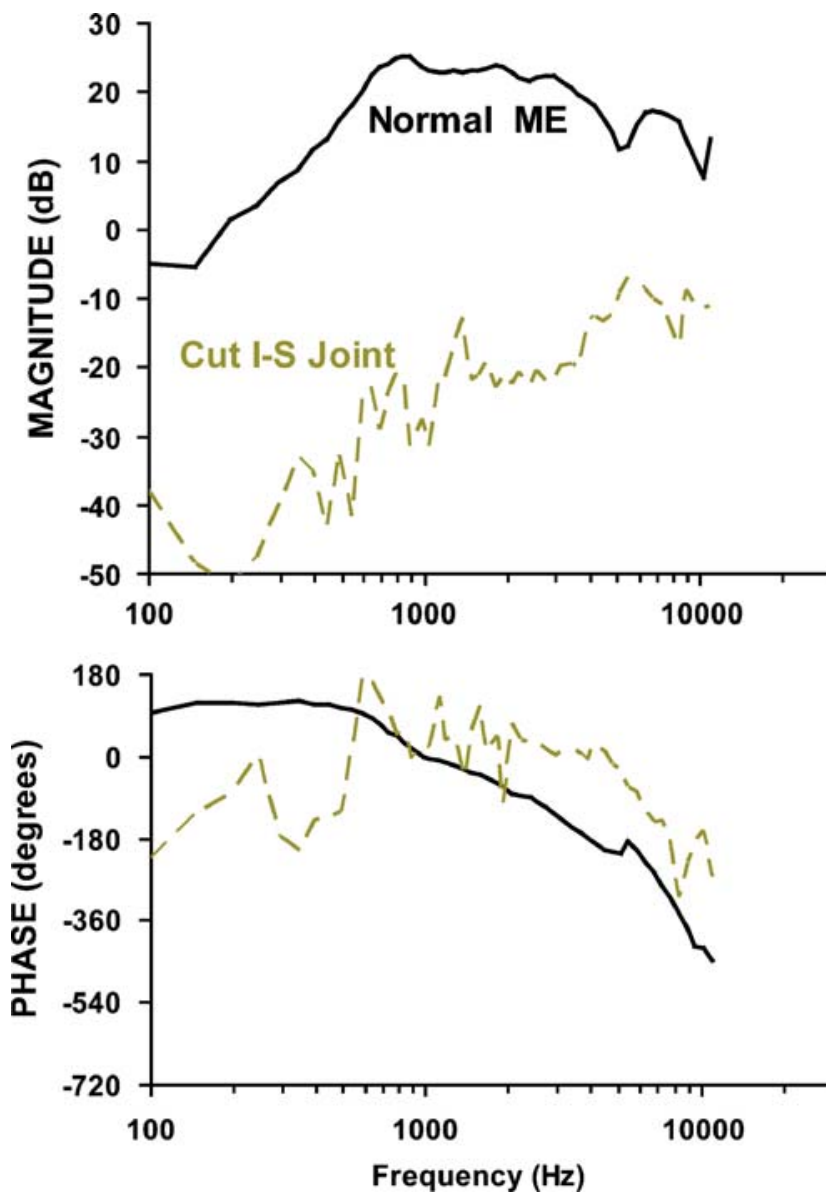

FIG. 8. Effect of ossicular discontinuity on the normalized differential pressure $\left(P_{\mathrm{SV}}-P_{\mathrm{ST}}\right) / P_{\mathrm{EC}}$. Disarticulation of the middle ear results in $30-50 \mathrm{~dB}$ decrease in the differential pressure, demonstrating the cancellation of similar high-frequency signals transmitted to both scalae (Fig. 9).

between previous measurements by Merchant et al. (1996, $n=1$ ) and Aibara et al. (2001, $n=12$ ), as shown in Figure 10. Aibara et al. (2001) "corrected" the stapes laser measurement by taking the cosine of the measurement angle with respect to a pistontype motion. However, some recent data suggest that such an angle correction is only valid at frequencies less than $1 \mathrm{kHz}$ where the piston-like component of stapes motion dominates, e.g., Chien et al. (2006). Others have also shown that at frequencies above $1 \mathrm{kHz}$, stapes motion is not a simple piston-type motion (Heiland et al. 1999; Hato et al. 2003; Decraemer and Khanna 2004). Therefore, we did not "correct" our stapes velocity measurements, and if Aibara et al. did not make the angle correction, their $\left|Z_{C}\right|$ would be slightly higher than what is plotted in Figure 10, close to our data. Merchant et al. (1996) estimated $Z_{C}$ by subtracting the measured impedance at the stapes with an intact cochlea by the impedance at the stapes with a fluid-drained cochlea. To measure impedance, they removed the incus from the stapes and stimulated the oval window of the stapes directly with sound. Stapes velocity was measured in the direction of the piston-type motion, using an optical lever. If instead they had made stapes motion measurement with an angle similar to ours and Aibara et al.'s, their low-frequency $\left|Z_{C}\right|$ would likely be slightly higher in magnitude compared that to Figure 10, resulting in even higher $\left|Z_{\mathbb{C}}\right|$ compared to ours. Estimates of $Z_{C}$ by all three methods have limitations in that the stapes with an intact middle ear chain does not naturally move in a purely piston-type motion above $1 \mathrm{kHz}$. To make improved estimates of $Z_{\mathrm{C}}$, one would have to consider all three-dimensional stapes motion that contributed to volume velocity at the stapes footplate.

Differential impedance across the cochlear partition $\left(Z_{\text {Diff }}\right)$

Assuming that the volume velocities are the same for the stapes and the round window, $U_{\mathrm{Stap}}=U_{\mathrm{RW}}$ (Stenfelt et al. 2004a, b), we can represent the cochlea with the
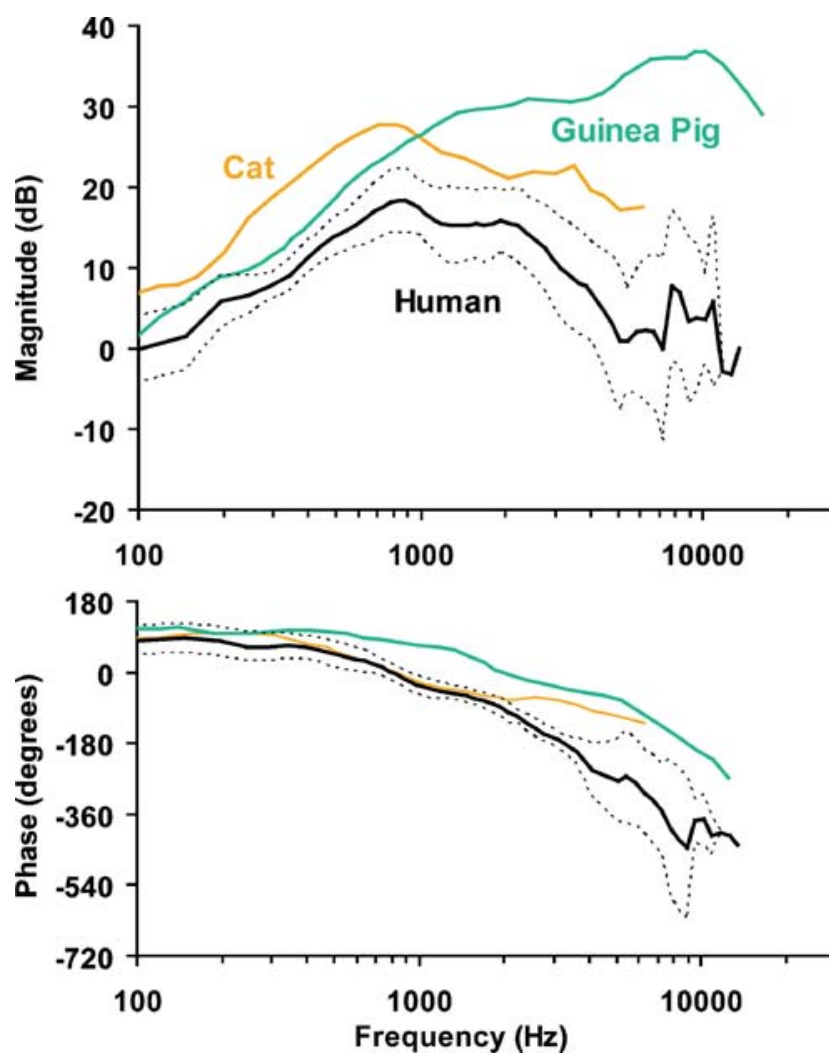

FIG. 9. The differential pressure, $\left(P_{\mathrm{SV}}-P_{\mathrm{ST}}\right) / P_{\mathrm{EC}}$, across the partition in humans (mean and standard deviation, $n=6$ ) is plotted with that of the cat (median, $n=6$, Nedzelnitsky 1980) and the guinea pig (mean, $n=5$, Dancer and Franke 1980). 

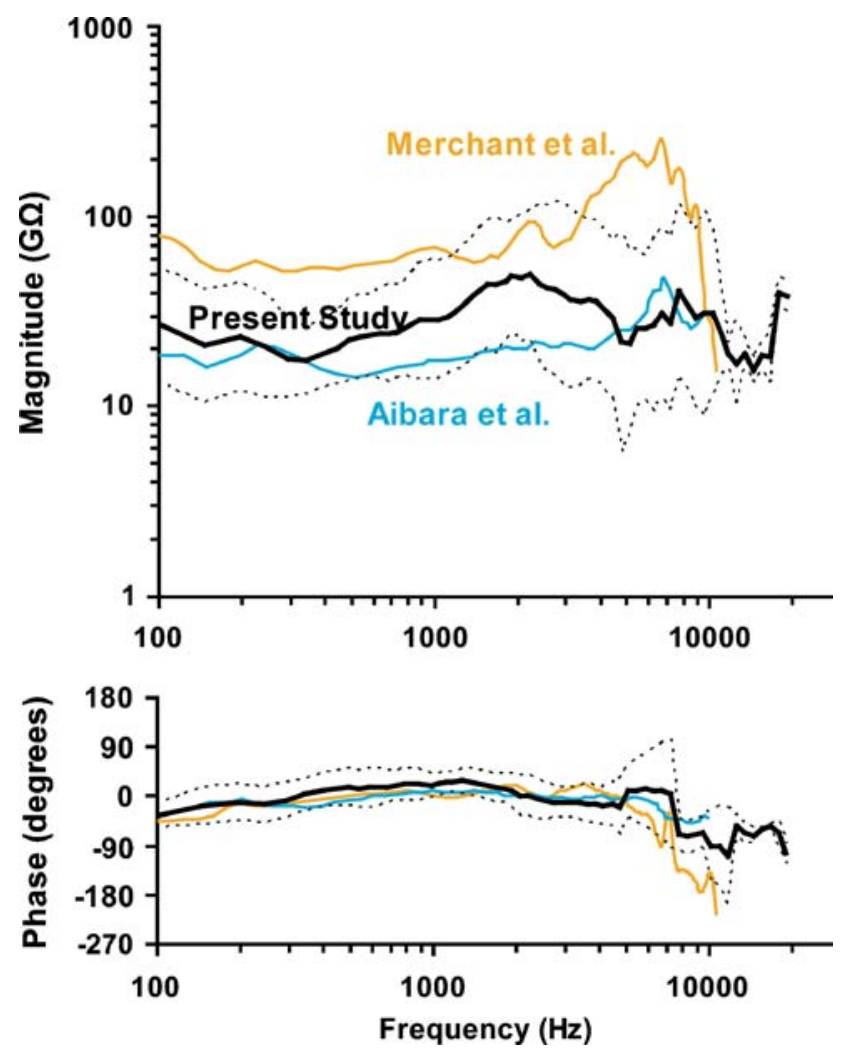

FIG. 10. Plot of the cochlear input impedance $\left(Z_{C}\right)$ with units of mks $\Omega\left(\mathrm{Pa} \mathrm{s} / \mathrm{m}^{3}\right)$. Our present study $(n=6)$ results in values of $Z_{\mathrm{C}}$ that generally fall between previous measurements by Aibara et al. (2001, $n=12$ ) and Merchant et al. (1996, $n=1)$.

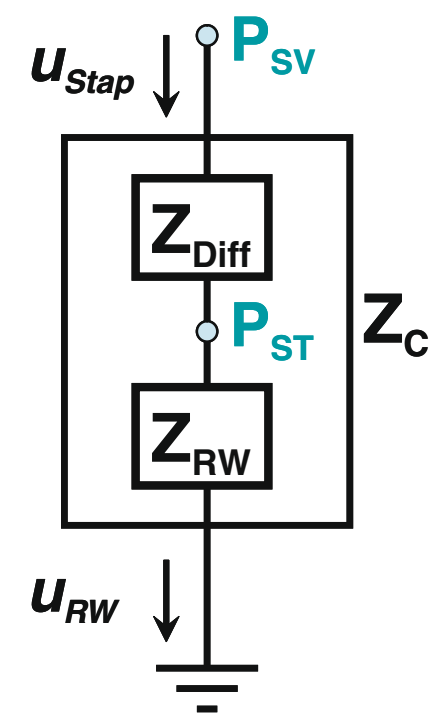

FIG. 11. The relationship of cochlear sound pressures and stapes volume velocity is described by this circuit. We assume that the volume velocities are the same for the stapes and the round window, $U_{\text {Stap }}=U_{\text {RW }}$. The cochlear input impedance $\left(Z_{C}\right)$ is the sum of the differential impedance $\left(Z_{\text {Diff }}\right)$ and the round window impedance $\left(Z_{R W}\right), Z_{C}=Z_{\text {Diff }}+Z_{R W}$. The ground connection at the bottom of the schematic represents the sound pressure in the middle ear cavity; we assume the pressure in the cavity is zero because in our preparation the middle ear cavity is widely opened to the atmosphere. circuit diagram in Figure 11 where $Z_{C}$ is the sum of the differential impedance $\left(Z_{\text {Diff }}\right)$ and the round window impedance $\left(Z_{\mathrm{RW}}\right)$ :

$$
Z_{C}=Z_{\text {Diff }}+Z_{R W} \text {. }
$$

Therefore, $Z_{\text {Diff }}=Z_{\mathrm{C}}-Z_{\mathrm{RW}}$ (described earlier by Geisler and Hubbard 1975), and to calculate $Z_{\text {Diff }}$ from our measurements, we use:

$$
Z_{\text {Diff }}=\left(P_{S V}-P_{S T}\right) / U_{\text {Stap }} .
$$

Thus, unlike $Z_{\mathrm{C}}$, which includes $Z_{\mathrm{RW}}, Z_{\mathrm{Diff}}$ is the impedance between scala vestibuli and scala tympani.

Figure 12 plots the differential impedance, $Z_{\text {Diff }}$. Below approximately $1 \mathrm{kHz}, \angle Z_{\text {Diff }}$ is near $0^{\circ}$ and $\left|Z_{\text {Diff }}\right|$ is nearly independent of frequency, consistent with an acoustic resistance. At higher frequencies, $\left|Z_{\text {Diff }}\right|$ fluctuates, likely due to the complex highfrequency three-dimensional motion of the stapes (Heiland et al. 1999; Hato et al. 2003; Decraemer and Khanna 2004). Above $4 \mathrm{kHz}$, the standard deviation increases due to the variability in $P_{\mathrm{ST}}$ measurements.

Compared to $Z_{\mathrm{C}}, Z_{\text {Diff }}$ magnitude has fewer fluctuations (independent of frequency) and the $Z_{\text {Diff }}$ phase is closer to $0^{\circ}$ for low frequencies. This indicates that $Z_{C}$ is being affected by the round window impedance, resulting in impedance that reflects both the effect of the resistance due to the differential impedance and compliance due to the round window impedance.
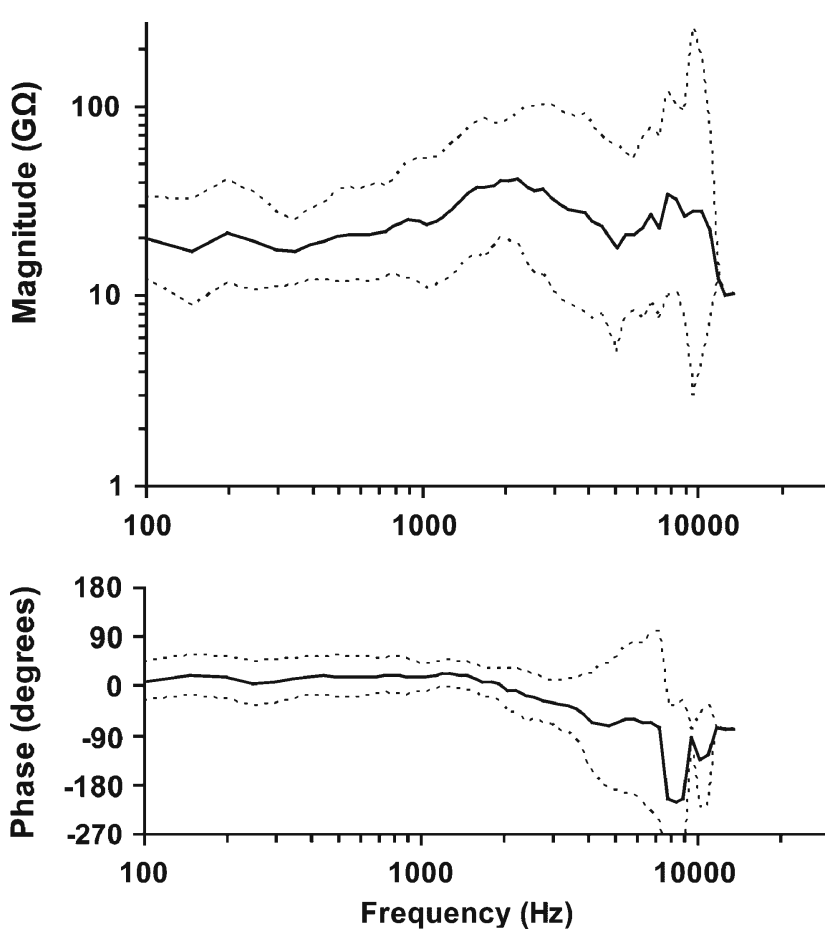

FIG. 12. Differential input impedance $\left(Z_{\text {Diff }}\right)$. For frequencies below $1 \mathrm{kHz}, Z_{\text {Diff }}$ is consistent with an acoustic resistance. 

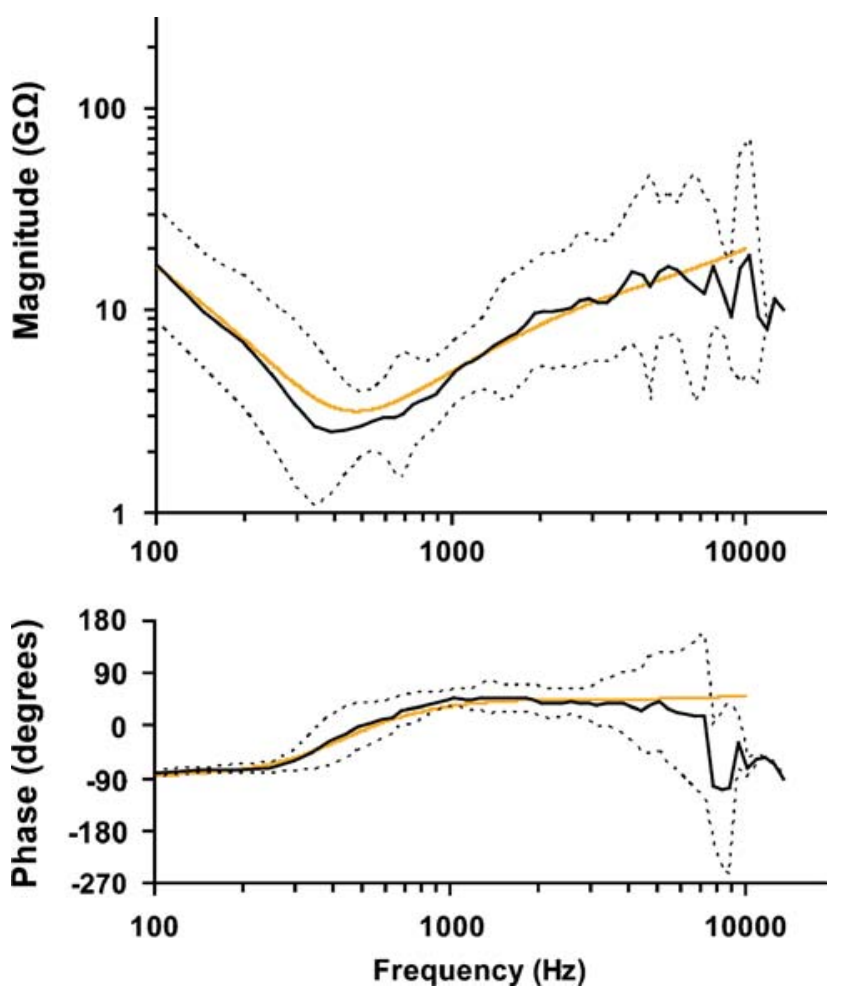

FIG. 13. Round window impedance, $Z_{\mathrm{RW}}=P_{\mathrm{ST}} / U_{\mathrm{Stap}}$ is plotted with a black line for the mean and dotted lines for the standard deviation. Below $300 \mathrm{~Hz}, Z_{\mathrm{RW}}$ behaves as a compliance. Above $300 \mathrm{~Hz}, Z_{\mathrm{RW}}$ becomes dominated by inertia and resistance. This behavior is consistent with distributed loss and inertia, wherein impedance increases as approximately the square root of frequency much as in a system with "skin effect." Behavior of a lumped parameter model for this system is plotted with gold-colored lines.

Therefore, we are able to separate the various impedances with our new measurements.

\section{Round window impedance $\left(Z_{\mathrm{RW}}\right)$}

The impedance of the round window can be expressed as

$$
Z_{R W}=P_{S T} / U_{S t a p}
$$

Figure 13 plots the round window impedance, $Z_{\mathrm{RW}}$. Below $300 \mathrm{~Hz},\left|Z_{\mathrm{RW}}\right|$ decreases about $-6 \mathrm{~dB} /$ octave and $\angle Z_{\mathrm{RW}}$ is near $-90^{\circ}$, consistent with the impedance being dominated by a compliance of approximately $9 \times 10^{-14} \mathrm{~m}^{3} / \mathrm{Pa}$. This value is similar to the compliance of the round window for the cat calculated by Lynch et al. (1982) and Nedzelnitsky (1980; $10 \times 10^{-14}$ and $16 \times 10^{-14} \mathrm{~m}^{3} / \mathrm{Pa}$, respectively). At higher frequencies, $\left|Z_{\mathrm{RW}}\right|$ increases approximately as a square root of frequency ( $3 \mathrm{~dB}$ /octave) with $\angle Z_{\mathrm{RW}}$ near $45^{\circ}$. It is clear that this behavior will not be well modeled with a simple lumped parameter model of conductance, inductance, and resistance. For example, Nedzelnitsky (1980) attempted to model similar $Z_{\mathrm{RW}}$ behavior in the cat, but as can be seen in Fig. 16 of Nedzelnitsky (1980), the phase of their measurement is near $45^{\circ}$ compared to their simple lumped parameter model going to $90^{\circ}$. Impedance characteristics that vary as the square root of frequency (i.e., magnitude as the square root of frequency and phase of $45^{\circ}$ ) or other sublinear functions of frequency are found in many distributed parameter systems, such as in the electrical impedance of conductors with "skin effect" (Dorf 1993; Sen and Wheeler 1998) and the acoustic impedance of oscillating gas flows in small tubes (Beranek 1954) and microchannels (Veijola 2001). The distributed parameter system characteristics of $Z_{\mathrm{RW}}$ are such that the effective resistance increases as the square root of frequency and the effective inductance/mass decreases as one over the square root of frequency. Therefore, it is reasonable to infer that this behavior is due to distributed inertia and energy dissipation of the cochlear fluids.

To model distributed parameter effects with lumped parameter circuit models, it is convenient to utilize Foster- or Cauer-form-iterated networks (Sen and Wheeler 1998; Veijola 2001; Roberge 1975). To model the round window impedance characteristic across frequency, we have used the circuit model of Figure 14, which includes a Foster-form-iterated network ( $N=6$ branches, with $X^{2}=10$ spacing $)$ to capture the effects above $300 \mathrm{~Hz}$. The lumped model thus uses six $(N)$ pole-zero pairs with pole-to-pole and zero-to-zero spacings of a factor of ten $\left(X^{2}\right)$ in frequency to approximate the behavior of the distributed system (which has infinite poles and zeros. (See Roberge 1975 for considerations in selecting order and spacings in such approximating networks.) The parameters used to model $Z_{\mathrm{RW}}$ are: $C=9 \times 10^{-14} \mathrm{~m}^{3} / \mathrm{Pa}$, $L=4.62 \times 10^{7} \mathrm{~kg} / \mathrm{m}^{4}, R=2.34 \times 10^{8} \mathrm{~Pa} \mathrm{~s} / \mathrm{m}^{3}, N=6, X=$ $10^{1 / 2}$. The behavior of this model is plotted with goldcolored lines in Figure 13, and it can be seen that the results closely match the experimental data. This modeling exercise shows that $Z_{\mathrm{RW}}$ has both distributed

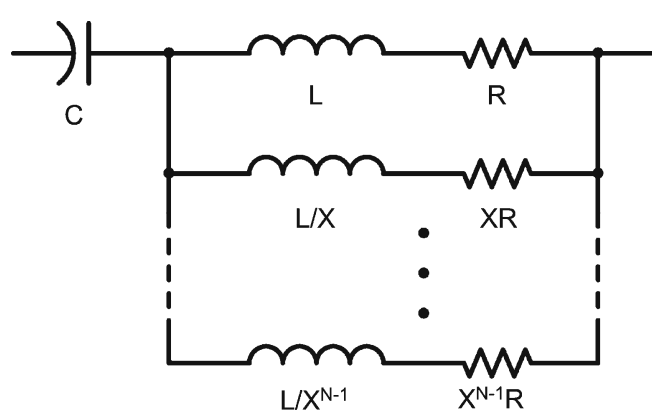

FIG. 14. Lumped parameter circuit model of the round window impedance. The model includes an iterated Foster network to model high-frequency effects. Model parameters are $C=9 \times 10^{-14} \mathrm{~m}^{3} / \mathrm{Pa}, L=$ $4.62 \times 10^{7} \mathrm{~kg} / \mathrm{m}^{4}, R=2.34 \times 10^{8} \mathrm{~Pa} \cdot \mathrm{s} / \mathrm{m}^{3}, \mathrm{~N}=6, X=10^{1 / 2}$. 
inertia and damping, that is, both the effective resistance and mass vary with frequency. However, this model is not unique in capturing this behavior nor do the parameters necessarily have physiological correlates. This model demonstrates that the observed behavior is consistent with varying resistance and mass across frequency, and that well-established models that have this behavior can match our data closely. What causes this behavior is speculative at present, but may reflect differences in fluid motion with frequency, as suggested by the different modes of round window motion observed across frequency (Khanna and Tonndorf 1971; Stenfelt et al. 2004a, b).

\section{CONCLUSIONS}

Sound pressure measurements, $P_{\mathrm{SV}}$ and $P_{\mathrm{ST}}$, were made simultaneously in the human cochlea with microscale fiberoptic pressure sensors. These sensors are extremely small and provide linear sensitivity and wide frequency response. However, they require detailed fabrication and are prone to sensitivity changes with mechanical perturbation. We only presented data that had pressure sensor calibration certainty to $2 \mathrm{~dB}$. Precautions were taken to ensure that air was not introduced into the cochlea and that the pressure sensors were sealed to their cochleostomies.

$P_{\mathrm{SV}}$ was significantly larger than $P_{\mathrm{ST}}$ for a wide frequency range, but both scala pressures were similar at low (below $300 \mathrm{~Hz}$ ) and high (above $5 \mathrm{kHz}$ ) frequencies. This shows that $P_{\mathrm{ST}}$ has a significant effect on the overall input to the cochlea at low and high frequencies. Phases were similar except at low frequencies (below $500 \mathrm{~Hz}$ ), where $\angle P_{\mathrm{SV}}$ showed approximately $1 / 8$ cycle lead while $\angle P_{\mathrm{ST}}$ slightly lagged near zero.

The middle ear gain, $P_{\mathrm{SV}} / P_{\mathrm{EC}}$, was bandpass with maximum of approximately $20 \mathrm{~dB}$ near $1 \mathrm{kHz}$. Below $1 \mathrm{kHz}$, the magnitude increased approximately $20 \mathrm{~dB} /$ decade with phase approximately $1 / 8$ cycle, indicating that elastic components of the middle ear dominate. The phase was linear with frequency above $1 \mathrm{kHz}$, with a group delay of approximately $83 \mu \mathrm{s}$. This $83-\mu$ s delay through the middle ear is over twice that found in the gerbil, reflecting significant inter-species difference in the middle ear.

Measuring $P_{\mathrm{SV}}$ and $P_{\mathrm{ST}}$ at the base simultaneously provides the differential input pressure at the base, which is the input to the cochlea that drives auditory transduction. The normalized differential input pressure $\left(P_{\mathrm{SV}}-P_{\mathrm{ST}}\right) / P_{\mathrm{EC}}$ had a bandpass character with peak magnitude of $10-28 \mathrm{~dB}$ around $1 \mathrm{kHz}$, similar to $P_{\mathrm{SV}} / P_{\mathrm{EC}}$ for mid-frequencies but affected by $P_{\mathrm{ST}}$ at low and high frequencies.
Disarticulating the middle ear showed that both $P_{\mathrm{SV}} / P_{\mathrm{EC}}$ and $P_{\mathrm{ST}} / P_{\mathrm{EC}}$ had similar high frequency sound pressures transmitted without the middle ear. However, because both scalae had similar highfrequency pressure signals, taking the difference caused near cancellation of these signals. Therefore, ossicular discontinuity resulted in a substantial decrease in the normalized differential input pressure, $\left(P_{\mathrm{SV}}-P_{\mathrm{ST}}\right) / P_{\mathrm{EC}}$, consistent with the hearing deficits observed clinically. This shows that the differential input pressure $\left(P_{\mathrm{SV}}-P_{\mathrm{ST}}\right)$ is a superior measure of sound transmission compared to $P_{\mathrm{SV}}$ alone.

By measuring stapes velocity concurrently, the cochlear input impedance $Z_{\mathrm{C}}=P_{\mathrm{SV}} / U_{\text {Stap }}$ was obtained. We also obtained the differential impedance across the partition, $Z_{\mathrm{Diff}}=\left(P_{\mathrm{SV}}-P_{\mathrm{ST}}\right) / U_{\mathrm{Stap}}$, as well as the round window impedance, $Z_{\mathrm{RW}}=P_{\mathrm{ST}} / U_{\mathrm{Stap}} . Z_{\mathrm{Diff}}$ was generally resistive and $Z_{\mathrm{RW}}$ was dominated by compliance at low frequency (below $300 \mathrm{~Hz}$ ) and had distributed inertia and damping at high frequency (above $500 \mathrm{~Hz}$ ). Differential intracochlear sound pressure measurements in human temporal bones now allow for the monitoring of the input signal to the cochlea. Pathology of the middle ear, such as ossicular discontinuity, showed effects on the differential input pressure similar to known effects on the audiograms in patients. Differential sound pressure measurements will enhance our understanding of various pathologies, beyond what is afforded by measurements of stapes velocity or scala vestibuli pressure alone. In future experiments, this technique will allow us to explore pathologies such as inner ear dehiscences, cochlear stimulations such as round widow stimulation and bone conduction, and the effects of various cochlear stimulation and prostheses on middle and inner ear pathologies.

\section{ACKNOWLEDGMENTS}

This work was carried out in part through the use of MIT's Microsystems Technology Laboratories and the help of Kurt Broderick. We thank William Peake for valuable discussions and Diane Jones, Michaël Slama, and Brian MacDonald for their generous efforts. This study was supported by grants R01 DC04798 and T32 DC00020 from the NIH. Wei Dong and Elizabeth Olson's time was supported by R01 DC003130. We also thank Mr. Axel Eliasen and Mr. Lakshmi Mittal for their support.

\section{REFERENCES}

Aibara R, Welsh JT, Puria S, Goode RL. Human middle-ear sound transfer function and cochlear input impedance. Hear. Res. 152:100-109, 2001.

Beranek LL. Acoustics. New York, McGraw-Hill, 1954.

Chien W, Ravicz ME, Merchant SN, Rosowski JJ. The effect of methodological differences in the measurement of stapes motion in live and cadaver ears. Audiol. Neuro-otol. 11:183-197, 2006. 
Colletti V, Soli SD, Carner M, Colletti L. Treatment of mixed hearing losses via implantation of a vibratory transducer on the round window. Int. J. Audiol. 45:600-608, 2006.

DanCER A, Franke R. Intracochlear sound pressure measurements in guinea pigs. Hear. Res. 2:191-205, 1980.

Decraemer WF, Khanna SM. Measurement, visualization and quantitative analysis of complete three-dimensional kinematical data sets of human and cat middle ear. In: Gyo K, Wada H, Hato N, Koike T (eds) Middle Ear Mechanics in Research and Otology. NJ, USA, World Scientific, pp. 3-10, 2004.

Dong W, Olson ES. Middle ear forward and reverse transfer function. J. Neurophysiol. 95:2951-2961, 2006.

Dorf RC. Electrical Engineering Handbook. Florida, CRC, p. 1219, 1993.

Geisler CD, Hubbard AE. The compatibility of various measurements on the ear as related by a simple model. Acoustica. 33:220-222, 1975.

Hato N, Stenfelt S, Goode RL. Three-dimensional stapes footplate motion in human temporal bones. Audiol. Neuro-otol. 8:140152, 2003.

Heiland Ke, Goode RL, Masanori A, Huber AM. A human temporal bone study of stapes footplate movement. Am. J. Otol. 20:81-86, 1999.

Khanna SM, Tonndorf J. The vibratory pattern of the round window membrane in cats. J. Acoust. Soc. Am. 50:1475-1483, 1971.

KringLebotn M. Equality of volume displacements in the inner ear. J. Acoust. Soc. Am. 98:192-196, 1995.

Lynch TJ, Nedzelnitsky V, Peake WT. Input impedance of the cochlea in cat. J. Acoust. Soc. Am. 72:108-130, 1982.

Merchant SN, Rosowski JJ. Auditory physiology (middle-ear mechanics). In: Gulya AJ, Glasscock ME III (eds) Surgery of the Ear. Hamilton Ontario, B.C. Decker, pp. 59-82, 2003.

Merchant SN, Rosowski JJ. Conductive hearing loss caused by thirdwindow lesions of the inner ear. Otol. Neurotol. 29:282-289, 2008.

Merchant SN, Ravicz ME, Rosowski JJ. Acoustic input impedance of the stapes and cochlea in human temporal bones. Hear. Res. 97:30-45, 1996.

NADOL JB. Techniques for human temporal bone removal: Information for the scientific community. Otolaryngol. Head Neck Surg. 115:298-305, 1996.

Nakajima HH, Ravicz ME, Merchant SN, Peake WT, Rosowski JJ. Experimental ossicular fixations and the middle ear's response to sound: evidence for a flexible ossicular chain. Hear. Res. 204:60-77, 2005.

NedZELNITSKY V. Sound pressure in the basal turn of the cat cochlea. J. Acoust. Soc. Am. 68:1676-1689, 1980.
OLson ES. Observing middle and inner ear mechanics with novel intracochlear pressure sensors. J. Acoust. Soc. Am. 103:34453463, 1998.

Olson ES. Direct measurement of intra-cochlear pressure waves. Nature. 402:526-529, 1999.

PURIA S. Measurements of human middle ear forward and reverse acoustics: implications for otoacoustic emission. J. Acoust. Soc. Am. 113:2773-2789, 2003.

Puria S, Allen JB. Measurements and model of the cat middle ear: evidence of tympanic membrane acoustic delay. J. Acoust. Soc. Am. 104:3463-3481, 1998.

Puria S, Peake WT, Rosowski JJ. Sound-pressure measurements in the cochlear vestibule of human-cadaver ears. J. Acoust. Soc. Am. 101:2754-2770, 1997.

Roberge JK. Operational Amplifiers Theory and Practice. New York, Wiley, 1975, Section 13.3.5.

Ruggero MA, Temchin AN. The roles of the external, middle and inner ears in determining the bandwidth of hearing. Proc. Nat. Acad. Sci. U. S. A. 99:13206-13210, 2002.

Schloss F, Strasberg M. Hydrophone calibration in a vibrating column of liquid. J. Acoust. Soc. Am. 34:958-960, 1962.

Sen BK, Wheeler RL. Skin effects for transmission line structures using generic SPICE circuit simulators. In: Proc. Electrical Performance of Electronic Packaging, IEEE 7th Topical Meeting. pp. 128-131, 1998.

Stenfelt S, Hato N, Goode RL. Fluid volume displacement at the oval and round windows with air and bone conduction stimulation. J. Acoust. Soc. Am. 115:797-812, 2004a.

Stenfelt S, Hato N, Goode RL. Round window membrane motion with air conduction and bone conduction stimulation. Hear. Res. 198:10-24, 2004b.

Vejola T. Acoustic impedance elements modeling oscillating gas flow in micro channels. In: Nanotech 2001 Vol. 1, Technical Proceedings of the 2001 International Conference on Modeling and Simulation of Microsystems. NSTI Publications, Taylor \& Francis, FL, pp. 96-99, 2001.

vON BÉKÉSY G. Experiments in Hearing. New York, McGraw-Hill, 1960.

Voss SE, Rosowski JJ, Peake WT. Is the pressure difference between the oval and round windows the effective acoustic stimulus for the cochlea. J. Acoust. Soc. Am. 100:1602-1616, 1996.

Willi UB, Ferrazzini MA, Huber AM. The incudo-malleolar joint and sound transmission loss. Hear. Res. 174:32-44, 2002.

ZwisLocki JJ. The role of the external and middle ear in sound transmission. In: Tower DB (ed) The Nervous System, Human Communication and its Disorders, vol. 3. New York, Raven, pp. 44-55, 1975. 